\title{
On-orbit validation of the Geolocation Accuracy of the GOES-16 Geostationary Lightning Mapper (GLM) Flashes using Ground-based Laser Beacons
}

\author{
Dennis Buechler*a, Thomas Varghese ${ }^{\mathrm{b}}$, Peter Armstrong ${ }^{\mathrm{c}}$, James Bremer ${ }^{\mathrm{d}}$, Rivers Lamb ${ }^{\mathrm{e}}$, Jon \\ Fulbright ${ }^{\mathrm{f}}$, Steve Goodman ${ }^{\mathrm{g}}$ \\ anniversity of Alabama Huntsville, 320 Sparkman Drive, Huntsville, AL, USA 35805; \\ ${ }^{\mathrm{b}}$ Cybioms Corporation, Rockville, MD, USA; \\ ${ }^{\mathrm{c}}$ MIT/Lincoln Laboratory, Lexington, MA, USA; \\ ${ }^{\mathrm{d} A S R C}$ Federal, USA; \\ 'NASA/GSFC, Greenbelt, MD, USA; \\ ${ }^{\mathrm{f}}$ Arctic Slope Tech Services, Beltsville, MD, USA; \\ 'Thunderbolt Global Analytics, USA
}

\begin{abstract}
As part of the geolocation accuracy assessment of lightning flashes detected by the Geostationary Lightning Mapper (GLM) on the GOES-16 and GOES-17 satellites (Geostationary Operational Environmental Satellite), two satellite laser ranging stations employed laser beacon systems to generate transient light pulses that simulate natural lightning around $777.4 \mathrm{~nm}$ to validate the pre-launch spec of $5 \mathrm{~km}$. The pulse width, repetition rate, wavelength, and power of the laserpulses were configured to produce sufficient instrument response to be detected as synthetic lightning events by the GLM instrument. During the testing period from April 2017 to January 2018, the laser systems illuminated the GOES-16 satellite to observe diurnal variation of the GLM system response, with particular emphasis on geolocation accuracy. The final GOES-16 laser beacon tests, which used the latest updates of the geolocation algorithms implemented by the GOES-R Ground Segment, showed the offsets between the GLM geolocated location and the known laser locations were within $5 \mathrm{~km}$.
\end{abstract}

Keywords: GOES, Geostationary Lightning Mapper, lightning, geolocation, laser, SLR, validation

\section{INTRODUCTION}

\subsection{GLM Overview}

The Geostationary Lightning Mapper (GLM) (Goodman et al, 2013) is a wholly new instrument onboard the NOAA (National Oceanic and Atmospheric Administration) GOES-16 and GOES-17 satellites, which were launched on November 19, 2016, and March 1, 2018, respectively. The GLM detects the optical signals at cloud top produced by lightning in real time, with the objective of improving high impact and severe weather warnings. Much of the cloud-tocloud lightning that precedes severe storms is not visible from the ground. The lightning associated with a given storm at any time of the day must be accurately geolocated to monitor that storm's intensity and evolution.

The GLM is a staring sensor having a circular field of view (FOV) with an $8^{\circ}$ radius, truncated in the N/S and E/W directions. From its post launch test (PLT) location at geostationary orbit and $89.5^{\circ} \mathrm{W}$ longitude, it observes all of the contiguous US (CONUS) and Central America, almost all of South America, and parts of southern Canada (See Figure 1).

*dennis.buechler@nasa.gov; phone 1 256-961-7827; fax 1 256-961-7979 


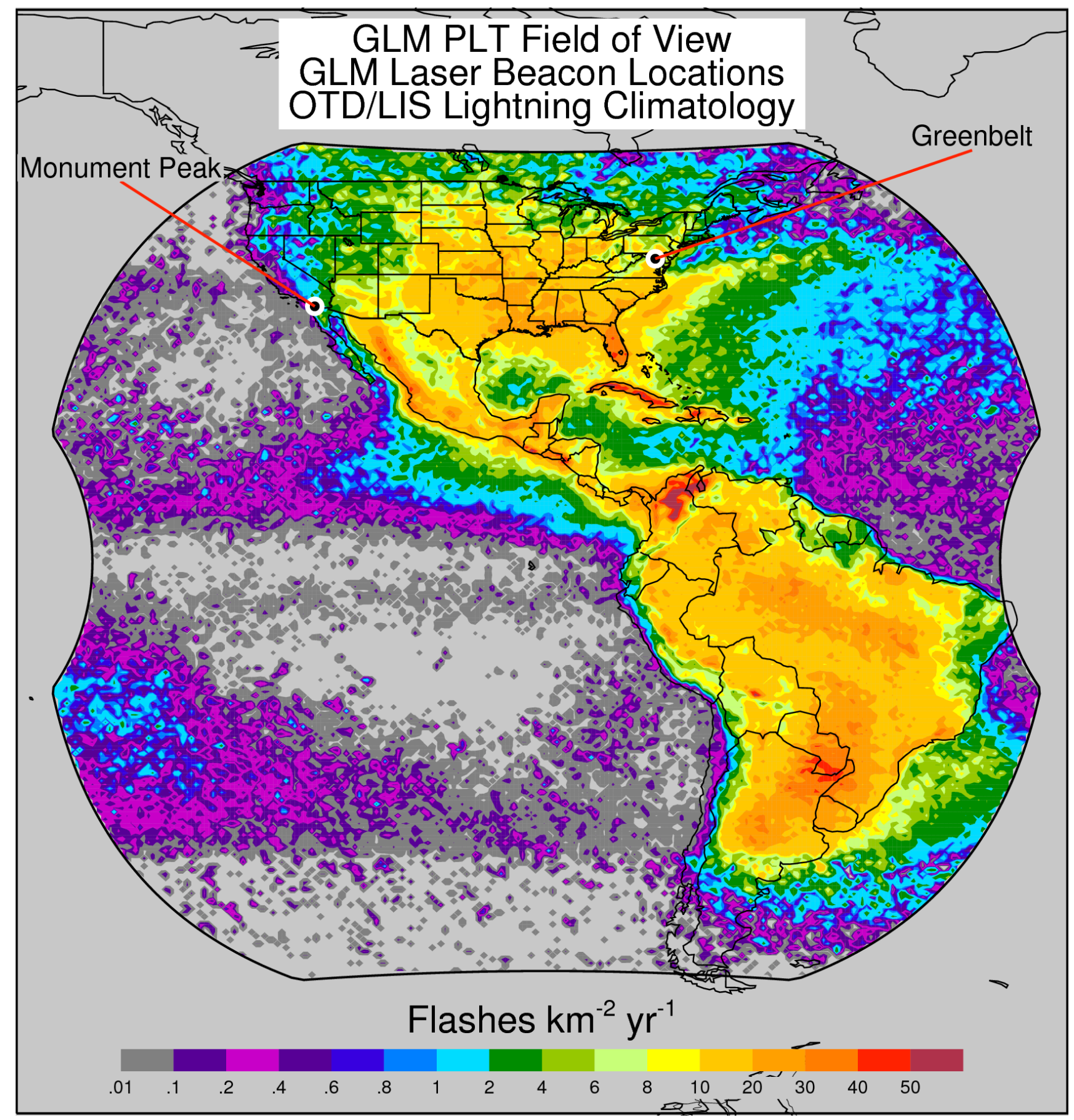

Figure 1. GLM field of view at $89.5^{\circ} \mathrm{W}$. The locations of the laser beacon sites are shown as white cross hairs. The lightning flash rate density values shown within the GLM FOV are from Optical Transient Detector ${ }^{2}$ (OTD) and LIS observations (1995-2013) ${ }^{3}$.

The GLM has a 1372x1300 Si CCD focal plane array ${ }^{4}$ operating at a $503 \mathrm{~Hz}$ frame rate. It has 30x30 $\mu \mathrm{m}$ pixels that subtend $8 \times 8 \mathrm{kms}$ at nadir, with a variable pitch CCD that decreases to $20 \mu \mathrm{m}$ with increasing field angle to reduce the off-nadir footprint growth. The GLM's nominal wavelength is $\lambda=777.4 \mathrm{~nm}$ with a bandwidth of $\Delta \lambda=1 \mathrm{~nm}$, to cover an oxygen triplet that is produced in the lightning's plasma. 
An onboard processor compares the signal in each pixel to an average of previous signals in that same pixel and declares a lightning event detection whenever the signal exceeds that average by more than a programmable threshold. This architecture allows the GLM to detect lightning even during the daytime against a solar-illuminated cloud when the lightning in a pixel is two orders of magnitude weaker than the background. The parameters of detected events are telemetered to the ground in real time. Software filters in the ground processing reject false alarms generated by natural phenomena, such as solar glints and linear streaks produced by solar radiation and cosmic rays. The GLM system declares a lightning flash when two or more events are detected in the same or adjacent pixels within a $1 / 3$ sec time window.

\subsection{Geolocation of Lightning}

The average background values from all pixels are transmitted to the ground every 2.5 minutes. These background values are used to form a background image that provides inputs to the coastline identification algorithm. The coastal boundaries are used to determine the residual offsets between geolocations of coastline pixels determined by ground processing and the known positions from a high-resolution coastal geography database. This comparison works with background images collected for approximately $4-6$ hours centered at satellite noon each day. All GLM detections are geolocated from the spacecraft orbit and attitude data, the temperatures of GLM structural and optical elements, and the compensation determined from the coastline analysis.

The GLM system geolocation error budget allocation is $140 \mu \mathrm{rad}(3 \sigma)$. The location accuracy depends on the thermal gradients on the instrument structure and the model of the cloud-top height. Thermal gradients alter the GLM instrument boresight, and the gradients have diurnal and seasonal variations due to the solar illumination of the instrument and the mounting platform to the spacecraft. Cloud top height is approximated using two parameters to describe a surface above the Earth ellipsoid, which provides a cloud-top height having a latitudinal dependence. The uncertainty in this approach increases with increasing field angle due to detection of light leaving from the sides of clouds instead of the cloud top. These parameters are determined using the comparison of GLM output to results from RF lightning detection networks. These comparisons are challenging because the RF detection systems and the GLM instrument detect different phenomena produced by lightning. Even though the cloud-top model is incorporated in the data products generated by the GLM ground system, the actual laser beacon light events from the SLR sites have extremely accurate geodetic positions. The ground system data products must be modified to account for the light originating from the SLR sites instead of the cloud-tops.

\section{LASER BEACONS}

\subsection{Satellite Laser Ranging (SLR) Ground Systems}

For validation of geolocation accuracy as part of the GLM's Post Launch Product Tests (PLPT), two laser beacons have been used to simulate lightning flashes detected by the $\mathrm{GLM}^{5}$. In contrast to natural lightning and coastline measurements, these laser beacons provide point sources of simulated lightning that originate from sites whose geodetic locations are known precisely and that can be transmitted at any time of the day when the line of sight (LOS) from the beacon to the satellite is clear.

We have been able to utilize the existing mobile laser (MOBLAS) stations with satellite laser ranging (SLR) capability that are maintained by NASA in support of the International Laser Ranging Service (ILRS). A long continental baseline within the GLM's FOV is implemented by installing one laser beacon in the MOBLAS 7 station (Figure 2) at Greenbelt, MD and another in the MOBLAS 4 station at Monument Peak, CA. The SLR site geodetic locations are known very precisely to millimeter levels of accuracy. The SLR transmit /receive optics is optimized for $532 \mathrm{~nm}$ and hence a wider FOV telescope co-aligned with fiber coupling optics tuned to near IR was piggy backed to the main telescope to support the laser beacon operations at $777.2 \mathrm{~nm}$. The pointing and tracking of the co-hosted beacon platform was managed by the servo-controlled SLR telescope and its software. The telescope assembly was bore-sighted with a radar and also equipped with a safety shutter to inhibit the laser beam upon an aircraft detection by the radar to prevent any accidental illumination of aircraft. 


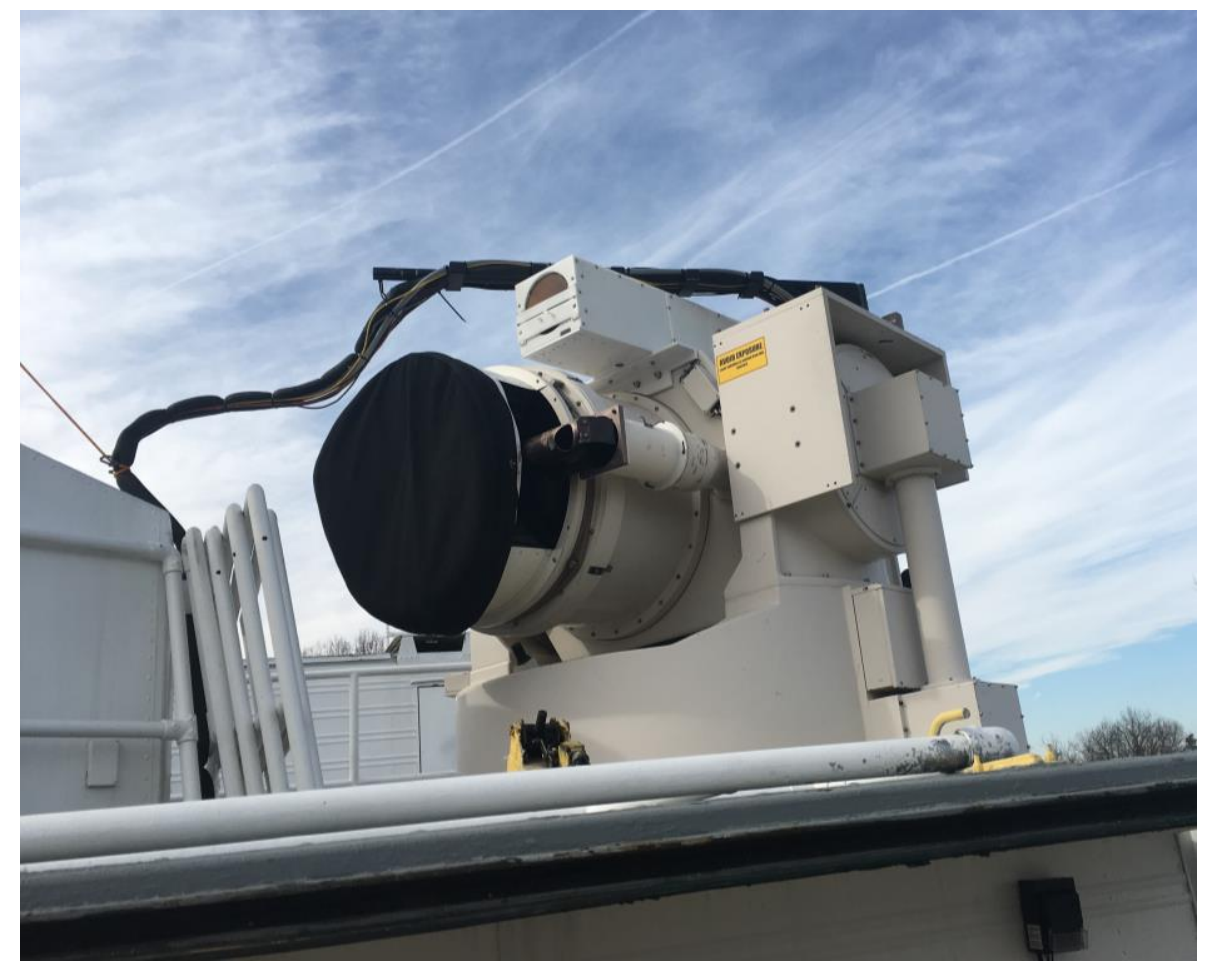

Figure 2. The piggybacked beacon telescope-fiber optics assembly on top of the NASA SLR telescope at the MOBLAS facility.

\subsection{Lasers}

Each beacon used a CW laser that is modulated to produce a pulse width of 1.5 milliseconds with a variable pulse repetition frequency (PRF). However, the operations were performed at either $50 \mathrm{~Hz}$ or $100 \mathrm{~Hz}$. The pulse width was chosen to maximize the energy received in a given GLM frame while avoiding excessive pulse splitting between consecutive frames. The PRF was high enough to produce multiple detections within the $1 / 3 \mathrm{sec}$ time window defining a lightning flash and yet low enough to allow the background to relax between events. The GLM's spectral bandpass decreases slightly with increasing field angle, so we operated both lasers at $777.2 \mathrm{~nm}$ to coincide with the GLM's peak transmission at the field angles where it observes the beacons. These parameters enabled the laser pulses to trigger detections by the GLM and to be identified as "true" lightning events by the ground processing filtering algorithms. The resulting GLM laser "lightning" geolocations can be compared to the precisely known locations of the laser beacon sites for validation. The laser did not interfere with the normal lightning detection of the GLM nor with the ABI because the beacon's wavelength lies between the ABI's Bands $2(0.64 \mu \mathrm{m})$ and $3(0.86 \mu \mathrm{m})$

\section{LASER BEACON OPERATIONS}

The laser beacon locations with respect to the GLM field of view are shown in Figure 1. The PLT laser tests were conducted both when the GOES-16 satellite was in post-launch test at $89.3^{\circ} \mathrm{W}$ longitude and after it was moved to its operational position as GOES-E at $75.2^{\circ} \mathrm{W}$ longitude.

Laser operations were scheduled at least a week in advance. Since the GLM is a staring sensor, we have considerable scheduling flexibility and can make the measurements that we require without interfering with the normal SLR operations. The weather forecast at each site was monitored in case the laser beacon test needed to be delayed due to weather conditions such as cloudy skies/or high winds. 
An ephemeris computed from the GPS data was used to determine the pointing of the lasers, and real time GLM data feedback was used to verify that the pointing is correct. The crews at the SLR sites can monitor these GLM observations of the beacon signals from a web site that displays this data with a latency of a 1-2 minutes. An example of the web display is shown in Figure 3. The GLM event energy, i.e. the net energy of (signal + background) - (estimated background) is divided by the energy of a single electron at $777 \mathrm{~nm}\left(2.56 \times 10^{-19} \mathrm{~J}\right)$ to estimate the number of photo electrons in each event. For comparison, Figure 4 shows GLM observations of actual lightning in the same web display format.
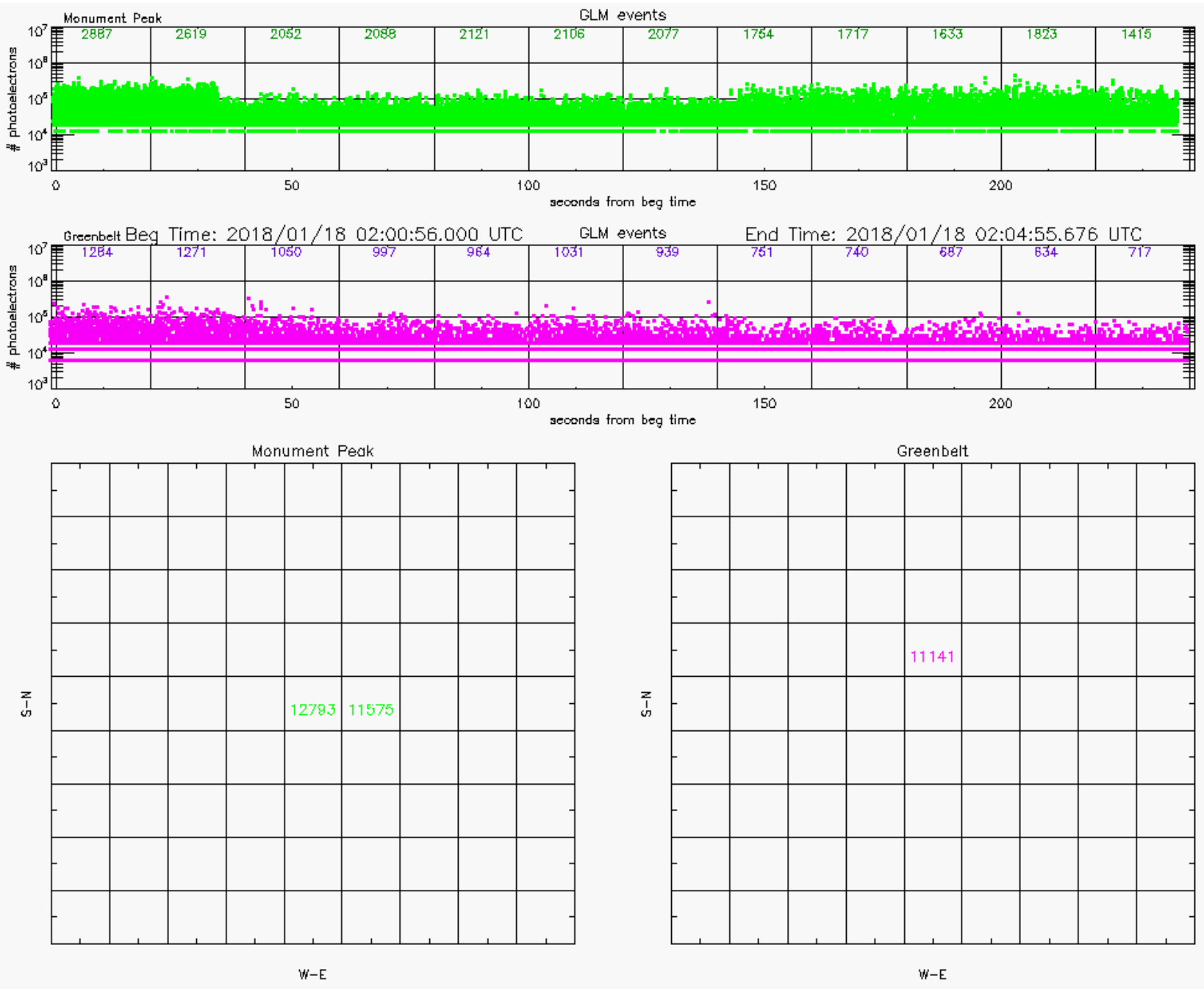

Figure 3. Web display of real time laser beacon observations by GLM. The bottom panels are 9x9 GLM pixels centered on the Monument Peak (left, green) and Greenbelt (right, magenta) SLR sites. The number of events in each pixel over a four minute period is shown if $>0$. The upper two panels are four minute time series of the event intensity in each 9x9 pixel region (with the number of events every $20 \mathrm{sec}$ plotted near the top of each panel).

\section{RESULTS}

Laser beacon tests for GOES-16 GLM were performed from March 2017 through January 2018. During this time the Ground Processing Algorithms (GPA) were updated a number of times to optimize the geolocation parameters for GLM geolocation. The final laser beacon tests were performed when the GOES-16 satellite was located in the GOES-East 
operational position at $75.2^{\circ} \mathrm{W}$ longitude and after the GPA geolocation parameters were optimized. The time series of the final laser beacon session for GOES-16 GLM is shown in Figure 5. The changes in event intensity were due to a deliberate effort to reduce the light intensity presented to the on-board sensor by applying beacon pointing angle offsets in azimuth and elevation to steer the beam away from the light collecting optics to better quantify detector performance. During the PLT, the CCD sensor exhibited intensity dependent overshoot effects that produced spurious detections in pixels adjacent to the actual signal. Since the beacons are point sources, we anticipated detections in at most a $2 \times 2$ block of pixels but observed detections in up to nine pixels. This beacon data was used to modify the algorithm and mitigate the problem. (Hardware changes will be implemented in the GLM's on GOES-T and U.)
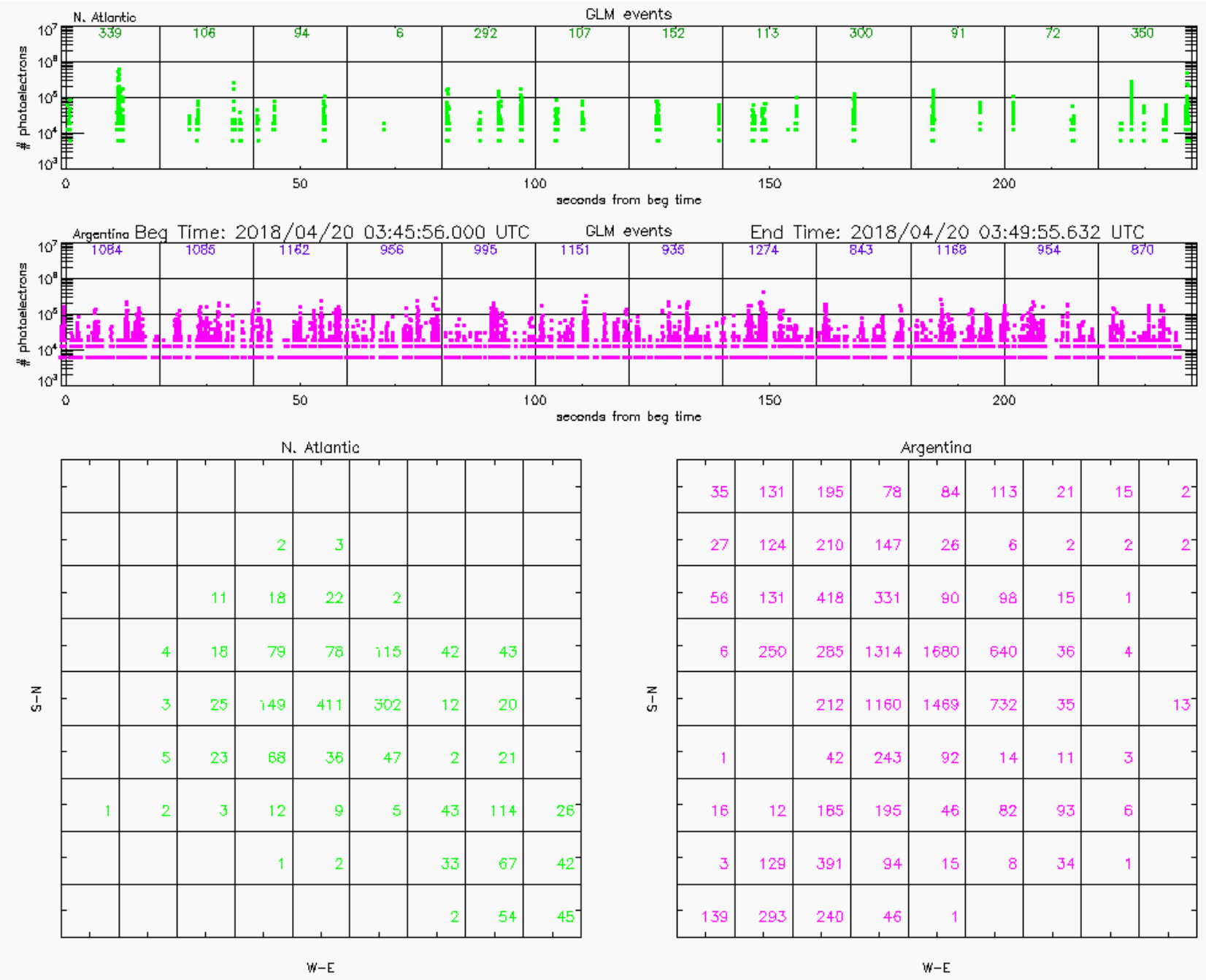

Figure 4. As in Figure 3 but for actual lightning. One storm was over the North Atlantic (green, top and left plots) and another was over Argentina (magenta, middle and right plots). 
The panels in Figure 6 shows the GLM laser 'flash' locations as geolocated by the GPA. The GPA geolocates the signals from GLM at cloud height (which varies according to latitude) instead of to the surface. Since the actual laser location of the laser is at the surface, we adjust the laser location to where the GPA would locate the beacon. This analysis compares the GPA-determined locations to the adjusted SLR location based on the local value of the cloud-top height. For this session both sites were within the requirements of $5 \mathrm{~km}$ (Monument Peak spatial offset $2.64 \mathrm{~km}$, GSFC offset $4.38 \mathrm{~km}$ ). There were two other laser beacon sessions while GOES-16 was at $75.2^{\circ} \mathrm{W}$ longitude and with optimized geolocation parameters. In both of these sessions the offset between the expected laser location and the GLM observed locations were also within $5 \mathrm{~km}$. This is within the GLM pixel size of 8-14 km.
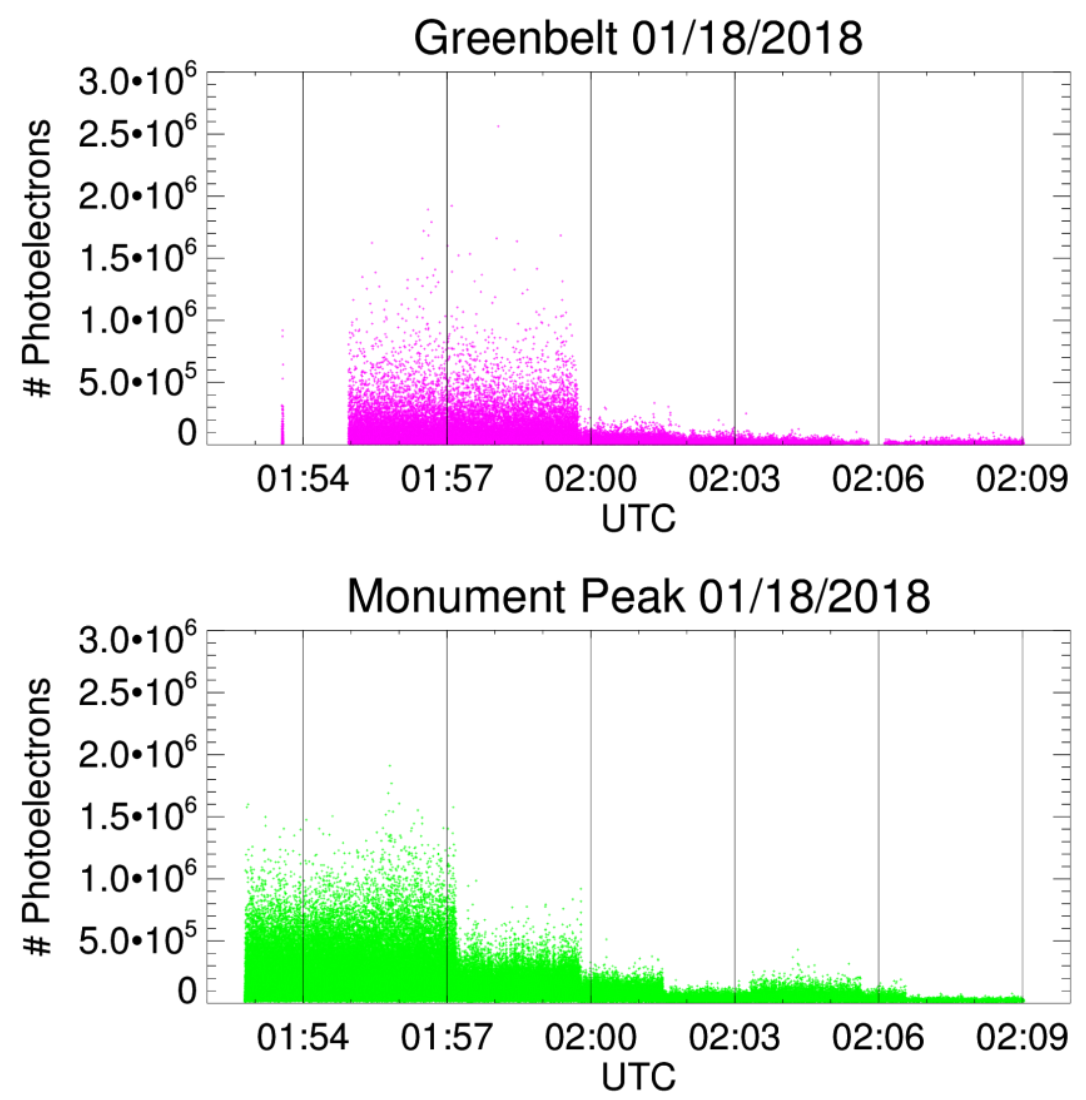

Figure 5. Time series of GLM observed event energy during the last GOES-16 laser beacon test. The upper panel is the Greenbelt laser (magenta) and the bottom panel is Monument Peak (green).

\section{SUMMARY AND CONCLUSIONS}

We have designed, developed, and operated two laser beacons at well characterized sites over a long baseline to verify the pre-launch predicted performance geolocation requirement of $5 \mathrm{~km}$. The transmitted laser pulses were detected and identified as lightning events by GLM, and they were used to assess the accuracy of the GLM's lightning geolocation. We developed a process to perform the laser beacon test, including a near real time feedback loop so the laser operators could determine that the laser beacon pointing was successful.

Results indicate that at the two laser beacon sites, the GLM geolocation algorithm locates the laser signal to within $5 \mathrm{~km}$ of the cloud top adjusted laser beacon location. The beacon measurements provide unambiguous control points throughout the diurnal cycle to verify the image navigation technique and the GLM's thermal/optical model or, if necessary modify them. Further analysis of the data is ongoing. In addition, similar tests are planned for the GLM on 
GOES-17. Meeting the geolocation specification may be more challenging at the GOES-W position because there will be far fewer coastline pixels within the GLM's FOV.
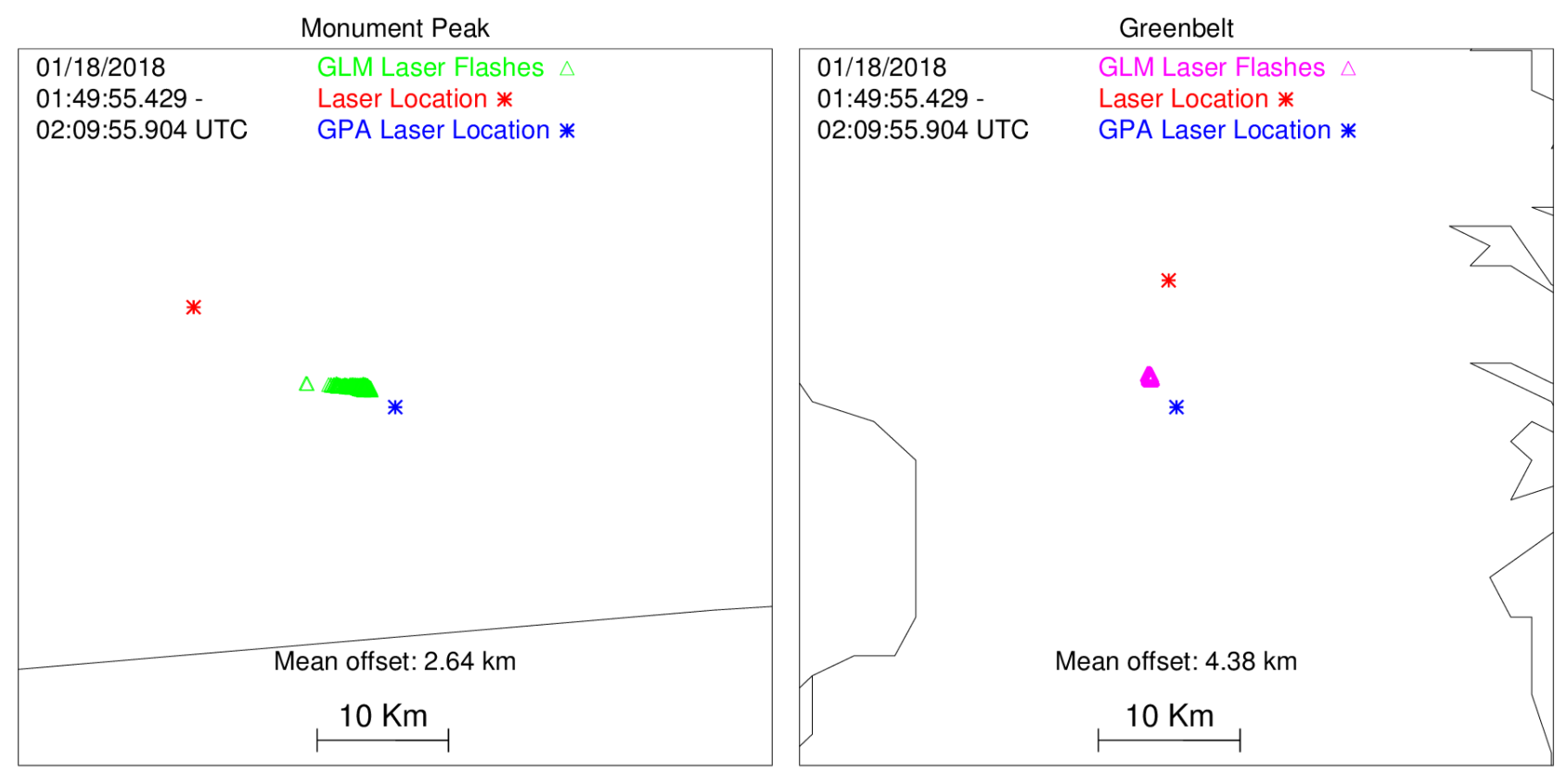

Figure 6. GLM flash locations of the laser beacon signal at Monument Peak (left) and Greenbelt (right). The actual laser location is denoted by the red asterisk in each plot. The GPA position is indicated by the cyan asterisk. The offsets shown are the distance between the mean flash location and the GPA location.

\section{ACKNOWLEDGEMENTS}

We would like to acknowledge David McCormick, Adam Milstein, and Donald Chu who have made significant contributions to the GLM laser beacon project.

\section{REFERENCES}

[1] Goodman, S. J., Blakeslee, R. J., Koshak, W. J., Mach, D., Bailey, J., Buechler, D., Carey, L., Schultz, C., Bateman, M., McCaul , E., Jr. and Stano, G., "The GOES-R Geostationary Lightning Mapper (GLM)," Atmos. Res., 125-126, 34-49 (2013).

[2] Christian, H. J. and Coauthors, "Global frequency and distribution of lightning as observed from space by the Optical Transient Detector,” J. Geophys. Res., 108, 119-149, (2003).

[3] Cecil, D. J., Buechler, D. E. and Blakeslee, R. J., "Gridded lightning climatology from TRMM-LIS and OTD: Dataset description," Atmos. Res., 135-136, 404-414 (2014).

[4] Bredthauer, R., Boggs, K., Bredthauer, G., Aamodt, E., Christian, H., Lesser, M., Gheno, K. and Reeve, B., "A novel CCD for application in high frame rate geostationary space based imaging," Proc. SPIE, 8453, 84531N (2012).

[5] Varghese, T., McCormick, D., Bremer, J., Buechler, D., Chu, D. and Goodman, S., "Enabling precise geo-spatial calibration of the GLM sensor on board the GOES-R satellite using ground-based Laser beacons from NASA Moblas 4 and 7," International SLR workshop, Potsdam, Germany, (2016). 